\title{
Note on a bubble model for excess electrons in liquid hydrocarbons
}

\author{
H. Hammer \\ Fachbereich Chemie, Universität Regensburg, Regensburg, Germany \\ W. Schoepe* and D. Weber \\ Fachbereich Physik, Universität Regensburg, Regensburg, Germany \\ (Received 22 July 1975)
}

In order to describe the mobilities of excess electrons in different liquid hydrocarbons a bubble model has been suggested. ${ }^{1}$ In that model the electron is considered to be localized in a cavity of radius $R$. The total energy $E_{t}$ of the bubble state is taken to be the sum of the quantum mechanical energy $E_{e}$ of the electron, the surface energy $E_{s}$ of the cavity, the usually negligible volume ene rgy (pressure times bubble volume), and the polarization energy $E_{\text {pol }}$. All of these energies depend on $R$.

For a stable bubble state to exist two criteria must be fulfilled:

(1) $E_{t}(R)$ must have a minimum at a stability radius $R=R_{0}$.

(2) $E_{t}\left(R_{0}\right)$ must be smaller than the lowest energy $V_{0}$ that a quasif ree electron can have in the particular liquid (edge of the conduction band).

This model was used earlier to describe the properties of electrons in liquid helium, neon, and hydrogen and stable bubble states were found for those liquids. ${ }^{2,3}$ Because of the low electron mobility in liquid hexane electron localization may also occur according to a bubble model. It is the purpose of the present work to show that using the macroscopic properties of liquid hexane measured at room temperature the above model does not yield a stable bubble state. However, we will also show that by assuming a higher dielectric constant the stability criteria can be met. The reason for this assumption will be given.

An electron in a cavity of radius $R$ in a polarizable medium of dielectric constant $\epsilon$ has a reduced energy (compared to the vacuum level) $E_{\mathrm{pol}}=-e^{2}(\epsilon-1) / 2 \epsilon R$. The cavity may be considered as a square-well potential of depth $V_{0}-E_{\mathrm{pol}}(R)$ in which the lowest ene rgy eigenvalue $E_{e}(R)$ can be computed numerically (Fig. 1). The surface energy $E_{s}(R)$ is given by $4 \pi R^{2} \sigma$, where $\sigma$ is the surface tension of the liquid.

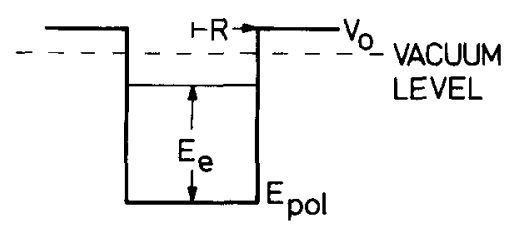

FIG. 1. Energy schematic of the localized electron. $V_{0}$ is the conduction band, $E_{\text {pol }}$ is the polarization energy, and $E_{e}$ is the lowest energy eigenvalue of the electron in the cavity.
Taking the macroscopic values of hexane $\sigma=20.4 \mathrm{erg} /$ $\mathrm{cm}^{2}$, and $\epsilon=1.89,{ }^{4}$ and $V_{0}=0.10 \mathrm{eV}$ as determined from work function measurements, ${ }^{5}$ we have computed

$$
E_{t}(R)=E_{e}(R)+E_{s}(R)+E_{\mathrm{pol}}(R)
$$

for $R$ ranging from 3 to $8 \AA$. The volume energy was neglected because it is smaller than $10^{-3} \mathrm{eV}$ in our range of interest.

As can be seen from Fig. 2 neither one of the two necessary criteria for a stable bubble state is fulfilled. ${ }^{6}$

The use of the macroscopic values for the surface tension and the dielectric constant, however, seems questionable at these small bubble radii.

In particular, the local dielectric constant in the vicinity of the bubble will be increased for the following reason. At short distances from a hexane molecule the $14 \mathrm{CH}$ dipoles do not cancel each other, i.e., the electron interacts with the individual dipoles and "sees" a polar hexane molecule. In the extreme case, one could consider the molecule to be broken up into independent $\mathrm{CH}$ dipoles. From Onsager's relation ${ }^{7}$ one can calculate the dielectric constant of such a hypothetical liquid. Taking the value of $0.4 \mathrm{D}$ for one $\mathrm{CH}$ dipole ${ }^{8}$ one obtains a dielectric constant $\epsilon=7,3$. We therefore found it interesting to vary the dielectric constant until stability of the bubble is reached and the known activation energy $E_{a}$ of the mobility is met (we the reby assume that the electron transport is a thermally activated process from the ground state inside an essentially immobile bubble into the highly mobile quasifree state: $\left.E_{a}=V_{0}+\left|E_{\mathrm{pol}}\right|-E_{e}\right)$. Our numerical results

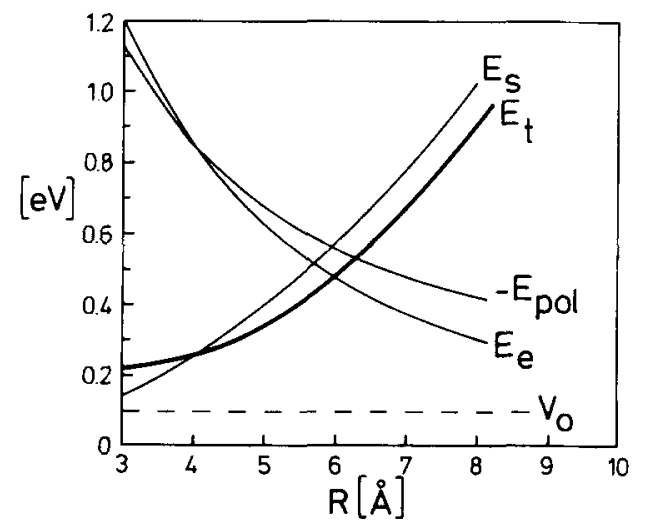

FIG. 2. Total energy $E_{t}$ of the electronic bubble vs bubble radius $R$ as computed from Eq. (1). Also shown are the other energy terms and $V_{0}$. 
TABLE I. Numerical results.

\begin{tabular}{lclll}
\hline \hline Liquid & $V_{0}(\mathrm{eV})$ & $\sigma\left(\mathrm{ergs} / \mathrm{cm}^{2}\right)$ & $E_{a}(\mathrm{eV})$ & resulting $\epsilon$ \\
\hline hexane & 0.10 & 20.4 & 0.19 & 2.6 \\
pentane & 0 & 15.9 & 0.18 & 2.9 \\
butane & -0.13 & 12.6 & 0.18 & 3.5 \\
propane & -0.13 & 15.0 & 0.13 & 3.1 \\
dimethyl- & & & & \\
butane & -0.15 & 18.7 & 0.05 & 2.9 \\
\hline
\end{tabular}

\section{are listed in Table I.}

The data for $V_{0}, \sigma$, and $E_{a}$ were compiled from the literature, ${ }^{9}$ ( $V_{0}$ of butane was a guess). The resulting $\epsilon$ are close to 3 , i. e. , about $50 \%$ higher than the macroscopic values. The stability radii we re all a round $3.5 \AA$.

In summary, we have shown that the simple bubble model can be applied to liquid hydrocarbons only if an inc reased dielectric constant due to not completely compensated dipoles is introduced. Clearly, a detailed microscopical model is needed which must take into account the interaction of the electron with the $\mathrm{CH}$ dipoles of the surrounding hydrocarbon molecules. The recent work of Feng et al. ${ }^{10}$ in which the interaction of the excess electron with various model $\mathrm{CH}$-dipole arrangements is treated seems to be a promising step in this direction.

We acknowledge valuable discussions with G. Bakale, K. Renk, R. Schiller, W. F. Schmidt, and B. Webster.

${ }^{*}$ To whom correspondence should be directed.

${ }^{1}$ R. Schiller, J. Chem. Phys. 57, 2222 (1972).

${ }^{2}$ B. E. Springett, M. H. Cohen, and J. Jortner, Phys. Rev. 159, 183 (1967).

${ }^{3}$ T. Miyakawa and D. L. Dexter, Phys. Rev. 184, 166 (1969) and references therein.

${ }^{4}$ The values for $\sigma$ and $\epsilon$ were taken from Beilsteins Handbuch der Organischen Chemie, 4th edition, 4th supplement, Vol. 1, Part 1, (Springer-Verlag, Heidelberg, 1972), p. 338.

${ }^{5}$ R. A. Holryod and R. L. Russel, J. Phys. Chem. 78, 2129 (1974).

${ }^{6} \mathrm{We}$ applied our numerical method also to the case of liquid neon where we found a stable bubble state in agreement with Ref. 3.

${ }^{7}$ L. Onsager, J. Am. Chem. Soc. 58, 1486 (1936).

${ }^{8}$ Landolt-Börnstein (Springer-Verlag, Berlin, 1951), Vol. 1, Part 3, p. 506.

${ }^{9}$ See, e.g., N. R. Kestner and J. Jortner, J. Chem. Phys. $59,26(1973)$ and references therein. The surface tensions were taken from Ref. 4.

${ }^{10}$ D. F. Feng, L. Kevan, and H. Yoshida, J. Chem. Phys. 61,4440 (1974).

\section{COMMENTS}

\section{Temperature effect on the absorption spectrum of $\mathrm{Eu}^{3+}$ in solution}

\section{Elhanan Würzberg}

Department of Physical Chemistry, The Hebrew University, Jerusalem, Israel (Received 25 August 1975)

The influence of solvents and anions on the absorption spectrum of $\mathrm{Eu}^{3+}$ is considerable in the case of the transition probability which may change up to an order of magnitude.$^{1-5}$ The energy of the transition changes very little.

The transitions ${ }^{7} F_{1}-{ }^{5} D_{0}$ and ${ }^{7} F_{0}-{ }^{5} D_{1}$ with $\Delta J=1$ are magnetic dipole transitions, ${ }^{5-8}$ the ${ }^{7} F_{0} \rightarrow{ }^{5} D_{0}$ and ${ }^{7} F_{1} \rightarrow{ }^{5} D_{1}$ transitions with $\Delta J=0$ are electric dipole transitions. The $J=0 \rightarrow J=0$ transition is a forbidden one ${ }^{8,9}$ and therefore very weak.

Red shifted by $300-400 \mathrm{~cm}^{-1}$ from the main absorption lines weaker and broader additional transitions were observed. ${ }^{1,3}$ Their origin has not been established with certainty. It was suggested that these are due to anion effects..$^{3.10}$ However, associated anions change the energy of these transitions only little. The energy separation between the ${ }^{7} F_{0}$ and ${ }^{7} F_{1}$ energy levels of $\mathrm{Eu}^{3+}$ in a solid matrix is $330 \mathrm{~cm}^{-1}$ in $\mathrm{Y}_{2} \mathrm{O}_{3},{ }^{11} 380 \mathrm{~cm}^{-1}$ in $\mathrm{LaCl}_{3},{ }^{12}$ in $\mathrm{EuCl}_{3} \cdot 6 \mathrm{H}_{2} \mathrm{O}$ it is $374 \mathrm{~cm}^{-1},{ }^{13}$ and in $\mathrm{LiNO}_{3}-$ $\mathrm{KNO}_{3} 320 \mathrm{~cm}^{-114}$ compared to $360 \mathrm{~cm}^{-1}$ in aqueous
$\mathrm{HClO}_{4}{ }^{15,16}$ The red shift in a solid matrix cannot be explained by the presence of nonionized salt as suggested by Chrysochoos ${ }^{3,10}$ but may be due to the ligand field. A similar phenomenon was found for other rare earths. ${ }^{17}$ Peacock ${ }^{18}$ suggested that the red shifted lines are the result of transitions from slightly higher levels, e.g., ${ }^{7} F_{1},{ }^{7} F_{2}$, thermally populated.

In an attempt to decide between these possibilities we measured the absorption spectrum as a function of temperature. Because of the very weak intensity of the transitions we used $1 \mathrm{M}$ aqueous $\mathrm{Eu}\left(\mathrm{ClO}_{4}\right)_{3}$ in a cell of $10 \mathrm{~cm}$ path length. Table I gives the calculated and experimental results for the area of the band, chasing bands with minimal overlap with other transitions.

The thermal population of the ${ }^{7} F_{0},{ }^{7} F_{1}$, and ${ }^{7} F_{2}$ levels can be calculated from

$$
n_{i} / n_{0}=\left(g_{i} / g_{0}\right) e^{-\Delta E / k T},
$$

$g_{0}$ and $g_{t}$ are the degeneracy of the levels. The degeneracy is given by $g=2 J+1$, where $J$ is the multiplicity. 